\title{
The Innovation of The Small Reconnaissance UAV Based on the Man-machine Engineering Machine Design
}

\author{
Weiqun $\mathrm{Cao}^{1, \text { a }}$, Lili Liu ${ }^{2, \mathrm{~b}^{*}}$, Jiaming Xue ${ }^{3, \mathrm{c}}$ \\ 1,2,3 Jiangsu Key Laboratory of Large Engineering Equipment Detection and Control \\ Xuzhou Institute of Technology \\ Xuzhou, Jiangsu Province, China \\ adxw89@sohu.com, ${ }^{b^{*}}$ dxwlll@sina.com. \\ 'XJM961224@.com
}

\begin{abstract}
With the development of science and technology, the science and technology used in military investigation and surveying and mapping are becoming more and more advanced, and small reconnaissance UAV gradually walked into people's lives. This paper mainly aimed at the improved design of the existing reconnaissance robots. It has improved the aesthetics and practicability of the small detection machine from the aspects of material, appearance, form, working method and manmachine interaction. The small surveillance aircraft can be used both on land and in the air, thus satisfying multi-disciplinary and multi-form work requirements.
\end{abstract}

Keywords—human factors engineering, innovation, scout plane, man-machine

\section{PRODUCT RESEARCH AND ANALYSIS}

Through investigation and analysis we can see that due to the particularity of users, they care more about small investigation UAV performance and environmental adaptability, which can meet the needs of normal operation under a variety of environment. Of course, they also hope the appearance can be improved a little bit. After all, the appearance of the existing small reconnaissance UAV on the market are relatively single, lack of innovation, difficult to meet the aesthetic needs of the future users[1]. In the final analysis, the designs of the existing products are lack of design and innovation.

Most of existing products use alloy material, which can't only ensure sufficient hardness, guarantee it will not be easily damaged in use. In addition, the weight of the alloy material has considerable advantages compared with the steel. But along with the deepening of the research progress and development of science and technology, the development of new materials allows reconnaissance robots to have a better choice. We aim to find out a material which can adapt to the future market in a better way to meet the needs of the consumers.

The battery life of existing small reconnaissance UAV is a big problem. But due to the particularity of its work, the small reconnaissance UAV is mainly suitable for short-range detection and reconnaissance. With the development of technology, a new type of battery is enough to meet the demand of its work, so currently we don't consider the power issue.

The biggest problem shown in the market investigation and analysis is small investigation UAV can't adapt to the operating environment very well. Once encountering with complex terrain or bad weather, it is hard for small investigation UAV to work properly. This is very fatal for user's experience. So how to improve the stability of small reconnaissance UAV to make it be able to work normally under different environments will be the top priority of this research project[2].

\section{The Main Problem of Existing Products}

Through the analysis of market research data, the usage of small reconnaissance UAV HAS the following problems: it can't be used in harsh environment, has single operating environment and poor appearance, etc. We hope to improve both appearance and function through the improved design this time. The combination of the ground investigation robot and the four axis aircraft can be used both on land and in the air and can satisfy the operating requirements of a variety of environments, and have certain solutions for complex environment. Regarding material and color, it can choose the new materials to improve its invisibility and stability. Hope that through several problems reflected in market research can be solved through this design, so as to meet the needs of the future market[3].

\section{DESIGN CONCEPTS}

Fully considering the problems shown in the market survey, this project will focus on how to improve the environment adaptability of small reconnaissance UAV, and improve the appearance of the existing product. Because it is small reconnaissance UAV, and according to the questionnaire it can be seen that it is mainly used on land and in the air, so we consider combining four axis aircraft and ground reconnaissance robot to adapt to the operation requirements of different environments[4].

Considering the four axis aircraft works by the lift force generated by four airscrews during the lifting, unlike the way of traditional aircraft, so the main body can use streamline 
design, which is not only beautiful, but also has good wind resistance in the flying process. Add the wheel to four axis aircraft in an innovative way to enable it to walk on the road for surveying and mapping. Considering issues that may happen when walking on the road such as collision, we can add guard bar to airscrew or conduct deformation design of four axis, so as to adapt to the operation requirements on land. Survey and mapping instruments such as cameras, infrared detector, night-vision device can add protection device to avoid its performance being influenced by long-term explosion outside.

Add a group of retractable wheels under the airscrews, of which the universal wheel direction is controlled by the engine of airscrews, and the motivation is controlled by another set of engine, namely each wing has two tiny engines, which can't only meet the needs of aerial reconnaissance, but also be able to meet the requirements of ground investigation, and the wheels also can be used as aircraft landing gear[5].

\section{A. Appearance modeling conception}

The appearance of current four axis aircrafts is relatively single. We hope to change that through this design. After full investigation and analysis, we decide to adopt the principle of bionic design on appearance design. The overall appearance is very much like the head of black mamba, and also a little bit like iron man's head. The hard appearance looks very tough, forming obvious contrast with the existing products. Wheels is extensible universal wheel, and can be fold up unnoticedly when flying; and put the wheels down when walking on the road, which can be used as both landing gear and ground investigation power. The front is replaceable camera, which can be changed to the corresponding detection equipment based on the actual demand. Add a "little tail" on the tail, it plays a significant role at the critical moment[6]. As shown in Fig. 1.

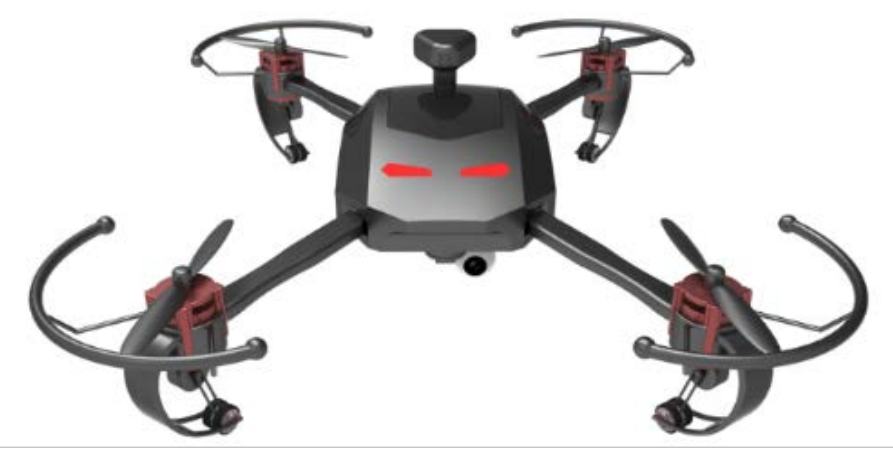

Fig. 1. Overall effect

\section{B. The design concept of aerial reconnaissance}

In aerial reconnaissance, universal wheel is fold up; the whole appearance is same as normal four axis aircraft. The front camera (can change to night vision at night) and secondary camera on the "tail" and infrared detectors installed on the four wings work together to conduct comprehensive investigation. As shown in Fig. 2.

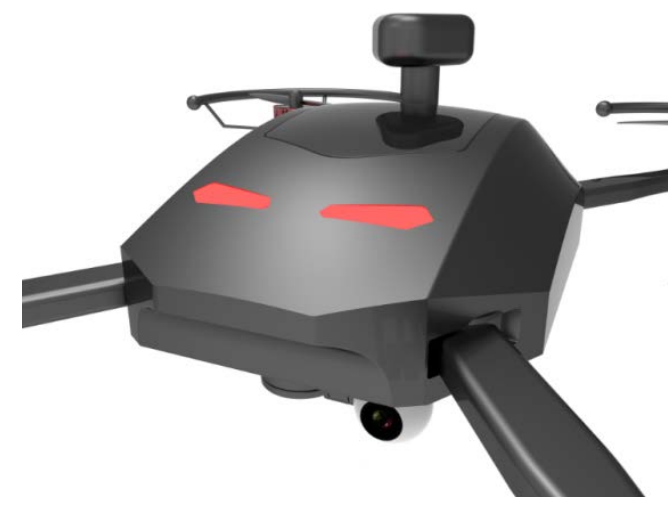

Fig. 2. Flight when rendering

\section{Design concept for ground reconnaissance}

In the ground reconnaissance, universal wheel is put down by a rotation shaft, which firstly acts as the landing gear, and then as the power device of walking on ground for ground investigation. The direction of wheel is controlled by the engine of airscrew, and the power is provided by another engine in the middle. The two engine work independently, not affecting each other. "Little tail" can lift, convenient for multiangle and comprehensive investigation.

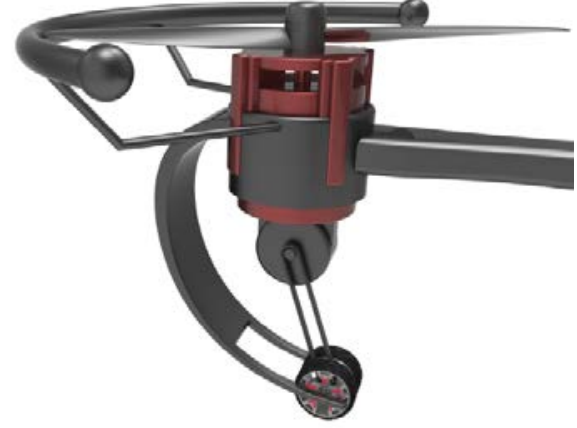

Fig. 3. The ground reconnaissance partial rendering

\section{The design concept for emergency investigation}

In case of emergency, "little tail" can fly out alone like a small rocket through its own reserves of energy, and conduct short term investigation which can be operated on the computer terminal. But this way of investigation can be used only once each time and "little tail" cannot be recycled. Due to its own limited reserves of energy, the time of short detection is relatively short, and the distance is also relatively short, suitable for investigation under emergency. 


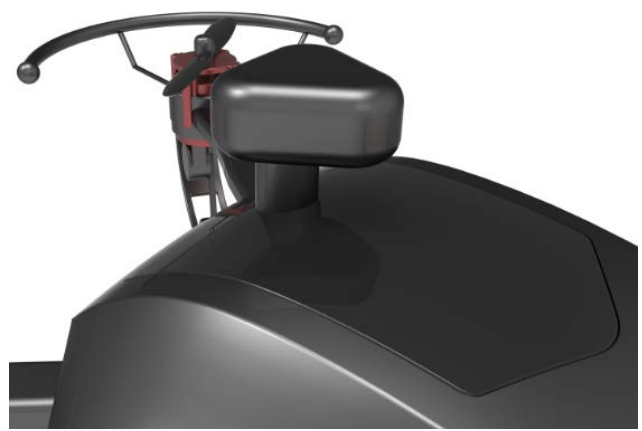

Fig. 4. "Little tail" details

\section{Human FACTOR ANALYSES}

\section{A. Color analysis}

Different colors bring people different intuitive feelings. For example, white represents purity, green represents youth and vitality, red represents joy. Medical devices mainly use gentle color to create a quiet atmosphere; danger sign should have bright color since sharp color contrast can cause people's attention; large machinery and equipment should use dark color to give people a kind of stable feeling.

The collocation of color is not following one's inclinations. The lively color doesn't have to be fancy. It requires comprehensive consideration of various factors. In the longterm practice, the use of the actual color should not only pay attention to aesthetics, but should pay more attention to practical performance. Use abstract form to express different feelings and different complex emotions, because color can change in three aspects, namely purity, lightness and hue. At the same time, the cold or warm sense of color will also have different effects, because different products have different functions, and people will have fixed impression for it. Products show some of its abstract forms through the collocation of color. Mastering the characteristics of color can conduct effective analysis and research of products. The connection between color and emotion should be mastered. Small smart detective robots main body is black or camouflage, main characteristic of which is easy to hide, and can well conceal itself and carry out investigation, at the same time also has fashion sense.

\section{B. Material analysis}

As a carrier of the products, materials have the same influence on products as color. Different material will bring different expressive force. All products require materials to express, because different materials have different aesthetic feeling. For example, wood materials can reflect human culture, metal materials generally gives people a sense of science and technology, plastic give people friendly feeling. Small smart reconnaissance robot choose a new type of radar detect material with light weight and hard, stiffness. It is very good at absorbing the sound waves generated by radar, which has anti-reconnaissance effect[7].

\section{The man-machine analysis}

The existing small scout planes all have automatic controller and navigation system. People can control it only through the remote control or remote computer. The simplification of human-computer interaction interface enables users to learn to use the small scout plane in the shortest time, which avoids the serious consequences that no one knows how to operate the plane in unexpected situations. The interaction interface, namely the design of computer terminal control interface, should be designed to be simple and clear. Simple and easy operation instruction allows more troops and geographical investigators to be familiar with the control of small scout plane in short time, so as to save time. In addition, judging from the size, the overall length of the fuselage is $500 \mathrm{~mm}$, width is $400 \mathrm{~mm}$, which is more reasonable. The small size is easy to carry. When encountering the narrow section and space, the fuselage can rotate to 400 $\mathrm{mm}$ width to pass more easily, not affecting the advance of the scout plane. As shown in Fig. 5. Unit: mm
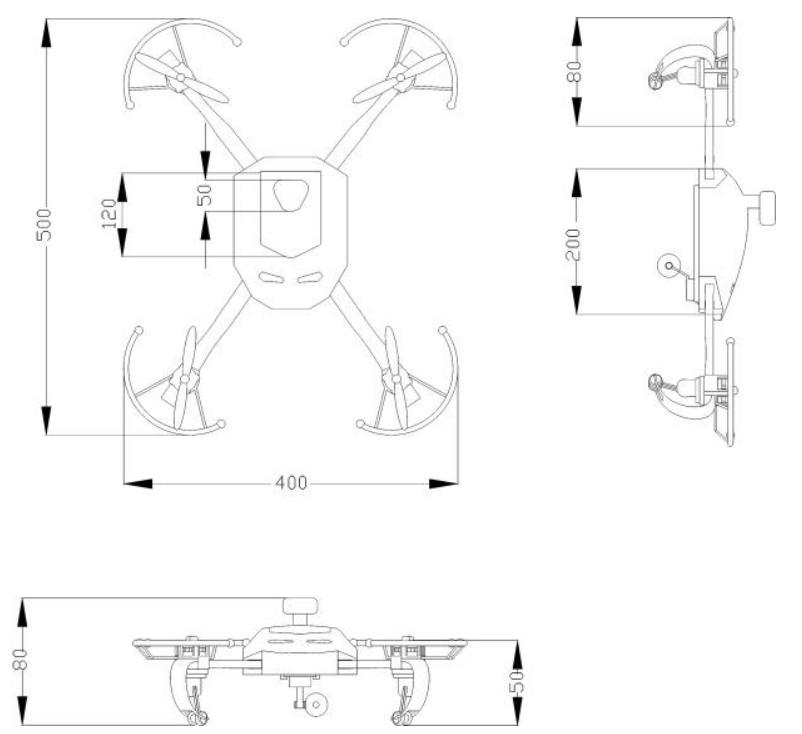

Fig. 5. Size chart

\section{CONCLUSIONS}

With the high-speed economic development today, the small scout plane is no longer military exclusive. Simple four axis aerial vehicle has gradually entered people's lives, but they are mainly used for military reconnaissance and topographic survey[8]. The existing small scout plane has single appearance; on function, it can only conduct short aerial reconnaissance, and can't be normally used under complex natural conditions. A new generation of small scout plane is developed which can work under various environments. It will gradually replace the existing small scout plane, and become the latest must-haves in the military and topographic survey fields.

This design takes full account of the shortcomings of the existing small scout plane, and finally determines the scheme after in-depth study and analysis. The small intelligent 
detection robot designed this time has achieved the design goal of air-ground dual use. In addition, it adds the emergency treatment measures design in an innovative way, which realizes the intellectualization of small scout plane in a real sense. The appearance uses the bionic design principle, abstracts the head outline of the Mamba, outlines the form with simple line, and matches it with the black appearance. It looks hale and solid, which is also a lot like the head of iron man. On function, the design of front replaceable came, innovative idea of "small tail "and structure design of universal wheel make the small scout plane more humanized.

\section{REFERENCES}

[1] Zheng Jianqi Li Xiang. Design methodology [M] Beijing: Tsinghua University Press, 2006.

[2] Wang Mingzhi. Product design[M] Beijing: China Youth Publishing House, 2002.

[3] (Germany) Bernhard .E Boolean Dirk industrial design: product modeling history Theory and Practice Hu Feize [J]. Beijing: China Building Industry Press, 2007.

[4] Ding Yulan ergonomics [M] Beijing: Beijing Institute of Technology Press, 2005.

[5] Wang Shenning, zhou Miing. The overall design of a portable surveillance plane[J]. The wind of science and technology, 2015 (9) :2727

[6] Ma Chong. Design and production of a flying wing model reconnaissance aircraft (bottom) [J]. Model airplane , 2011 (9) :34-37

[7] Liang Yi,Fan Yunxun . Design of the operation and protection system of lawn mower based on ergonomics. Machinery Design and Manufacture[J] . 2005 (12) :32-34

[8] Fang Rujing. Analysis of the aerodynamic characteristics of micro unmanned reconnaissance aircraft. University of north , 2014 